\title{
Clinical and Neuroimaging Correlates of Mild Cognitive Impairment in a Middle-Aged Community Sample: The Personality and Total Health through Life 60+ Study
}

\author{
Rajeev Kumar ${ }^{a, b}$ Ruth A. Parslow ${ }^{b}$ Anthony F. Jorm ${ }^{b}$ \\ Stephen J. Rosenman ${ }^{\mathrm{b}}$ Jerome Maller $^{\mathrm{b}}$ Chantal Meslin $^{\mathrm{b}}$ Kaarin J. Anstey ${ }^{\mathrm{b}}$ \\ Helen Christensen ${ }^{\mathrm{b}}$ Perminder S. Sachdev ${ }^{\mathrm{c}, \mathrm{d}}$ \\ ${ }^{a}$ Department of Psychological Medicine, Australian National University Medical School, ${ }^{\mathrm{b}}$ Centre for Mental \\ Health Research, Australian National University, Canberra, ${ }^{c}$ School of Psychiatry, University of New South \\ Wales, and ${ }^{d}$ Neuropsychiatric Institute, Prince of Wales Hospital, Sydney, Australia
}

\section{Key Words}

Mild cognitive impairment $\cdot$ Magnetic resonance

imaging $\cdot$ Brain atrophy $\cdot$ White matter hyperintensities

\begin{abstract}
This cross-sectional study aimed at determining the clinical and structural brain magnetic resonance imaging correlates of mild cognitive impairment (MCl). The data presented here are from the first wave of the longitudinal Personality and Total Health through Life $60+$ project. 2,551 community-dwelling individuals in the age range of 60-64 years were recruited randomly through the electoral roll. They were screened using Mini-Mental State Examination and a short cognitive battery. Those who screened positive underwent detailed medical and neuropsychological assessments. Of the 224 subjects who screened positive, 117 underwent a detailed assessment. Twenty-nine subjects fulfilled the Mayo Clinic criteria for $\mathrm{MCl}$. Magnetic resonance imaging scans were analyzed for 26 subjects with $\mathrm{MCl}$ as well as normal controls. Subjects were clinically evaluated for depressive symptoms and major and minor depression syndromes. Logistic regression analysis was performed predicting
\end{abstract}

$\mathrm{MCl}$ from anterior and mid-ventricular brain ratios, cortical atrophy measures, hippocampal volumes, volumes of amygdala and white matter hyperintensities after adjusting for age, gender, years of education, depression and physical disability. None of the neuroanatomical substrates appeared as predictors of $\mathrm{MCl}$. The only predictors were higher depression scores and fewer years of education. Structural neuroimaging may not have an added advantage in the detection of $\mathrm{MCl}$ in middle-aged community-dwelling subjects. It may be that this age group is too young for such brain changes to be identified.

Copyright (C) 2006 S. Karger AG, Basel

\section{Introduction}

There has been much recent interest in the category of mild cognitive impairment (MCI) as a possible transitional state between normal ageing and mild dementia [1]. It is estimated that about $10-15 \%$ of MCI subjects will progress to dementia per year compared to $1-2 \%$ normal controls [2]. This makes MCI an important target for intervention studies for the prevention or delaying of

\section{KARGER \\ Fax +4161306 1234 E-Mail karger@karger.ch} www.karger.com
C 2006 S. Karger AG, Basel $1420-8008 / 06 / 0211-0044 \$ 23.50 / 0$

Accessible online at: www.karger.com/dem
R. Kumar

Department of Psychological Medicine, The Canberra Hospital

PO Box 11

Woden ACT 2606 (Australia)

Tel. +61 2 62443204, Fax +612 62443228, E-mail Rajeev.Kumar@act.gov.au 
onset of dementia. MCI, however, remains a heterogeneous entity with no universally accepted definition and operational criteria [3]. Its stability as a diagnosis has also been questioned [4]. One approach to examining the validity of the diagnosis of MCI using proposed criteria is to examine its clinical and neuroimaging correlates and contrasting it with a cognitively normal control group.

A number of clinical and neuroimaging correlates of MCI have been reported in the literature. In previous studies, it has been reported that depression, apathy, irritability and suspiciousness have been associated with MCI [5-7]. It can also be argued that since MCI is conceptualized as a pre-dementia state, factors associated with mild Alzheimer's disease are also likely to be linked with MCI. Studies examining the neuroimaging markers have predominantly used structural magnetic resonance imaging (MRI), although functional neuroimaging has also been of interest $[8,9]$. Atrophy of medial temporal lobe structures such as hippocampus, amygdala or entorhinal cortex has been a focus of attention along with reduced intracranial volume (ICV), reduced total brain volume, atrophy of corpus callosum and increased white matter lesions [10].

Most studies of MCI have been conducted in clinic or patient samples, with only a few exceptions [11-12]. This sampling strategy runs the risk of biasing the sample because of the self-selection and the likely inclusion of subjects who are at the threshold of a diagnosis of dementia. We report the study of a randomly selected sample from the community, thus greatly reducing this bias. Furthermore, we examined a relatively younger group - aged 60-64 years - which was much less likely to be misdiagnosed with early dementia. We examined important clinical and MRI variables, with the following hypotheses: clinical: MCI is associated with (1) higher scores of depression and (2) fewer years of education; neuroimagingrelated: MCI is associated with (1) reduced hippocampal and amygdala volumes, (2) increased regional cortical and subcortical atrophy and (3) higher loads of white matter hyperintensities (WMHs).

\section{Methods}

\section{Subjects}

The sample was drawn from the Personality and Total Health (PATH) through Life Project designed to study the risk and protection factors for normal aging, dementia and other neuropsychiatric disorders. The study cohort comprised 2,551 individuals who were residents of the city of Canberra and the adjacent town of Queanbeyan, Australia. They had a mean of 13.72 years of education and were recruited randomly through the electoral roll. Enrolment to vote is compulsory for Australian citizens. The subjects were all community dwelling and none were from health facilities, such as hospitals or nursing homes. The response rate was $58.3 \%$ for the total sample. A subgroup of subjects that formed the health and memory substudy were drawn from the above sample. The first wave of this longitudinal study, data from which are being reported here, was conducted in 2001 and 2002 with a view to having the second assessment 4 years later. Approval for the study was obtained from the Ethics Committees of the Australian National University and University of New South Wales.

\section{Health and Memory Substudy}

Phase 1 (Screening). From the total study population of 2,551, subjects were selected for further examination if they had any one of the following: (1) a score of Mini-Mental State Examination [13] $\leq 25$, (2) a score below 5 th percentile on immediate or delayed recall of the California Verbal Learning Test [14] (immediate score of $<4$ or delayed of $<2$ ) or (3) a score below the 5 th percentile on either of the following two tests: Symbol-Digit Modalities Test [15] $(<33)$ or Purdue Pegboard [16] with both hands $(<8)$ or reaction time [17] (third set of 20 trials) $(>0.31 \mathrm{~s})$.

Phase 2 (Medical Examination and Consensus Diagnosis). Subjects who screened positive and consented to participate further were given a Structured Clinical Interview for Dementia (available from the authors) by one of two physicians. Whenever possible, we interviewed a close informant. We gathered information on medical history related to cognitive functioning, duration of such symptoms, history of other medical illnesses, diagnoses made by family medical practioners, current treatments, family history and psychiatric history. Self-report measures of depression and anxiety were available for the whole study population. A detailed physical examination was conducted with a particular focus on measurements of blood pressure and a neurological examination. Subjects who received a MCI-related diagnosis were referred to their family doctor for laboratory investigations, with the recommended tests being blood chemistry screen, glucose, thyroid functions, $\mathrm{B}_{12}$, complete blood counts, cholesterol and triglycerides. If it was indicated, we also recommended ESR, chest X-ray, ECG and EEG. MRI brain scans were performed on all subjects as part of the research protocol.

A battery of neuropsychological tests was administered covering frontal executive functions (Trails A and B, Verbal fluency and Clock drawing) [18-20]; language (Boston naming of 15 items) [21], constructional praxis of CERAD [22], memory (Rey Auditory-Verbal Learning Test with verbal recall and recognition) [23], recall of constructional praxis from CERAD for nonverbal memory, apraxia and agnosia [22]. The Clinical Dementia Rating Scale was also administered [24].

The diagnosis of MCI was made by consensus clinical judgement rather than by an algorithm. This is in contrast with previous algorithmic approaches used in population samples. The two medical practioners who did the assessment and two practicing psychiatrists with strong research interest in dementia disorders met periodically to discuss the assessments in detail, and arrive at diagnoses. Using checklists and information from the original cognitive data and the subsequent neuropsychiatric battery, we applied the diagnostic criteria proposed by Petersen et al. [2] (table 1) for MCI amnestic type. Prevalence of MCI in the PATH study has been reported previously [25]. 
Table 1. Diagnostic criteria for MCI amnestic type

1 Memory complaint preferably corroborated by an informant

2 Objective memory impairment (measured by memory performance score 2 or below on delayed California Verbal Learning Test)

3 Normal general cognitive function (MMSE 26 or above and overall IQ not significantly affected)

$4 \quad$ Intact activities of daily living

\section{Assessment of Depression Symptoms and Syndrome}

The Goldberg Depression Scale [26] was used to measure symptoms of depression. In addition, participants completed the depression section of the PRIME-MD Patient Health Questionnaire, which asks about DSM-IV Major Depression symptoms in the last 2 weeks [27]. This questionnaire was scored to give a continuous scale from 0 to 27 and also classify participants as having a Major or Minor Depressive Syndrome.

\section{Assessment of Physical Disability}

Physical disability was assessed using the Physical Component Summary of the 12-item Short Form Health Survey [28]. The other measures of physical health were presence of hypertension, diabetes, heart trouble or head injury. The apolipoprotein E4 status and homocysteine levels were available for the whole sample, but unfortunately we did not have adequate numbers with these measures to analyze their association with MCI.

\section{MRI: Subject Selection}

About 1 subject in 5 was selected at random for participation in the MRI study. Of 622 participants so approached, $478(77 \%)$ $($ men $=252)$ agreed to undergo an MRI brain scan, and provided written informed consent. This subgroup also provided a blood sample. Those who said during the initial interview that they were unwilling to have a scan were significantly $(\mathrm{p}<0.05)$ more likely to be female, of non-English speaking background, were less educated, with poor physical health and lower cognitive test scores. Those who refused to undergo a scan when actually invited were significantly more likely to be of non-English speaking background, were less educated, with poor physical health and lower cognitive scores. There was no significant difference in depression at either stage, although there was a trend towards fewer depressive symptoms in those who accepted at the second stage. In addition, MRI scans were performed in a subgroup of subjects who were part of a substudy of MCI. Of the 117 subjects who were screened for the substudy, 89 subjects completed MRI scanning.

\section{MRI Scans Protocol}

All subjects were imaged with a 1.5-tesla Philips Gyroscan scanner for $\mathrm{T}_{1}$-weighted $3 \mathrm{D}$ structural and $\mathrm{T}_{2}$-weighted FLAIR sequence MRI. A scout mid-sagittal cut for AC-PC plane alignment was first acquired. The $3 \mathrm{D}$ structural MRI was acquired in coronal orientation using a $\mathrm{T}_{1}$-weighted FFE sequence with the following parameters: $\mathrm{TR} / \mathrm{TE}=28.05 / 2.64$; flip angle $=30$; matrix size $=256$ $\times 256 ; \mathrm{FOV}=260 \times 260 \mathrm{~mm}$; slice thickness $=2.0 \mathrm{~mm}$ and inter- slice distance $=1.0 \mathrm{~mm}$, yielding over-contiguous coronal slices and an in-plane spatial resolution of $1.016 \times 1.016 \mathrm{~mm} /$ pixel. The FLAIR sequence was acquired in coronal orientation with TR/TE/ $\mathrm{TI}=11,000 / 140 / 2,600 ;$ matrix size $=256 \times 256 ; \mathrm{FOV}=230 \times$ $230 \mathrm{~mm}$; slice thickness $=4.0 \mathrm{~mm}$ with no gap between slices and in-plane spatial resolution of $0.898 \times 0.898 \mathrm{~mm} /$ pixel.

\section{Image Analysis}

MRI scans were transferred to an independent Windows NT workstation and analyzed using the software packages ANALYZE (Mayo Foundation, Rochester, Mich., USA) and SPM99 (Cognitive Neuroscience Group, National Hospital for Nervous Diseases, London). The anterior and mid-ventricular ventricle-to-brain ratios and visual cortical atrophy (frontal, mid-temporal and midparietal) ratings were completed for all subjects including MCI and healthy using the method of Victoroff et al. [29]. High intra-rater and inter-rater reliability (intra-class correlation coefficients $>9$ ) were also established for each of these measures. The hippocampus and amygdala volumes were traced manually using an adaptation of the method of Watson et al. [30]. High intra-rater and interrater reliability (intra-class correlation coefficients $>0.7$ ) were established for each of the above measures. The absolute volume of hippocampus was determined, and this was normalized for each subject's ICV. The ICV was computed automatically using an algorithm within SPM. WMHs were identified on FLAIR sequences and visually rated by 2 independent clinicians experienced in examining MRI scans on a modified Fazekas scale [31]. The interrater $(n=57$, weighted kappas for periventricular, deep white matter and deep gray matter regions were $0.82,0.90$ and 0.93 , respectively) and intra-rater $(\mathrm{n}=29$, rater 1 weighted kappas for periventricular, deep white matter and deep gray matter regions were $0.88,0.85$ and 0.93 and for rater $2,0.85,0.82$ and 0.84 , respectively) reliability has been excellent.

\section{Statistical Analysis}

Initial descriptive analysis involved comparing means and percentages (as appropriate) of sociodemographic, physical, depression, cognitive and brain measures. Then analyses were done splitting the data by gender. Predictors of MCI were evaluated using logistic regression, with odds ratio used as the index of effect size. For MRI measures, each one was entered individually and adjusted for age, gender, education, presence of depression and physical disability. The $\mathrm{p}<0.05$ significance level was used. Hippocampal and amygdala volumes were normalized by dividing these with ICV. We also analyzed the raw volumes and used ICV as a covariate in the regression analysis to see whether the results were any different.

\section{Results}

Figure 1 contains the flow chart showing the formation of study population. There were 224 subjects who met the screening criteria to participate in the study, and we interviewed 117. Of the 117 subjects who underwent detailed medical and neuropsychological examinations, 29 met the MCI diagnostic criteria set by Petersen et al. [2]. 


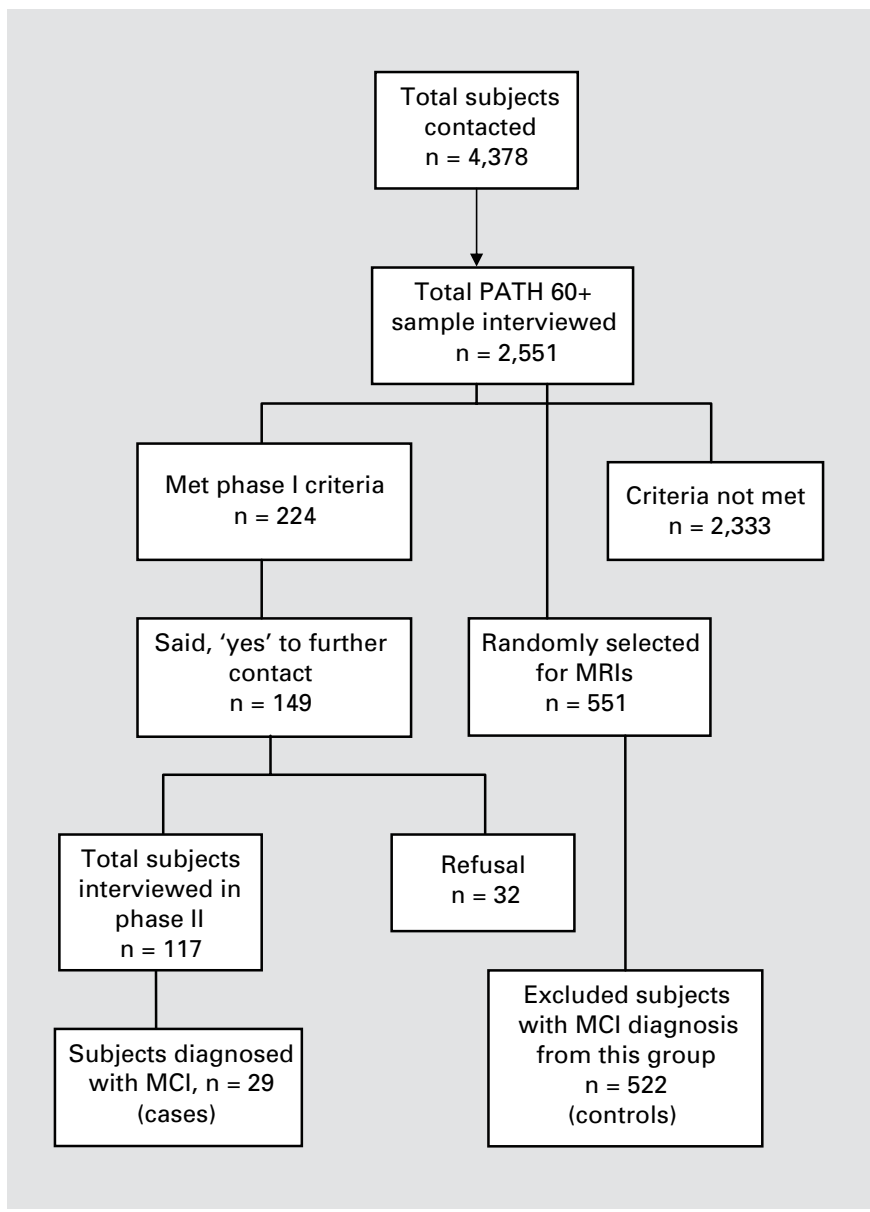

Fig. 1. Flow chart showing the formation of study population.
We then drew a sample of 522 subjects from those who were randomly selected for MRIs $(n=551)$ to act as controls by excluding those who received an MCI diagnosis.

The descriptive characteristics of the MCI and the non-MCI sample are given in table 2. Subjects with MCI had fewer years of education and higher depression scores compared with healthy controls. They were not different in terms of age, gender, physical health or other physical measures.

Table 3 shows the cognitive screening test scores between the MCI group and controls. Significant differences between groups were observed for all cognitive screening measures except choice reaction time.

None of the structural MRI measures were significantly different between groups. In regard to gender difference, no statistically different results were found in any of the above measures except women had significantly higher depression scores.

Logistic regression predicting MCI showed that lower years of education (OR 0.77, CI 0.67-0.89, p = 0.00) and high depression score (OR 1.21, CI 1.01-1.44, $\mathrm{p}=0.03$ ) were strong predictors of MCI after controlling for age, gender and physical disability (table 4). We further analyzed the association with depression using the DSM-IV diagnoses derived from the Patient Health Questionnaire and found that a diagnosis of minor depression was significantly associated with MCI $(17.2 \%$ in MCI versus $3.5 \%$ in non-MCI, Fisher's exact test, $\mathrm{p}=0.01$ ). Table 5

Table 2. Descriptive characteristics of the sample $(n=551)$

\begin{tabular}{|c|c|c|c|c|c|c|c|}
\hline & \multicolumn{3}{|c|}{ Non-MCI } & \multicolumn{3}{|c|}{ MCI } & \multirow[t]{2}{*}{$\mathrm{p}$} \\
\hline & $\mathrm{n}$ & mean $(\mathrm{SD})$ & $\%$ & $\mathrm{n}$ & mean $(\mathrm{SD})$ & $\%$ & \\
\hline \multicolumn{8}{|c|}{ Sociodemographic variables } \\
\hline Age, years & 522 & $62.55(1.46)$ & & 29 & $62.65(1.36)$ & & 0.71 \\
\hline Males & 276 & & 52.9 & 18 & & 62.1 & 0.33 \\
\hline Females & 246 & & 47.1 & 11 & & 37.9 & \\
\hline Education, years & 520 & $13.94(2.67)$ & & 29 & $11.93(2.99)$ & & 0.00 \\
\hline \multicolumn{8}{|l|}{ Health risk factors } \\
\hline SF-12 physical health & 518 & $49.65(8.81)$ & & 29 & $46.65(7.99)$ & & 0.07 \\
\hline Hypertension, definite & 522 & & 41.37 & 29 & & 37.93 & 0.71 \\
\hline Hypertension, probable & 522 & & 23.37 & 29 & & 34.48 & 0.17 \\
\hline Normotensive & 522 & & 35.24 & 29 & & 27.58 & 0.40 \\
\hline Diabetes, definite & 522 & & 8.04 & 29 & & 10.34 & 0.66 \\
\hline Stroke, self-reported & 520 & & 4.40 & 29 & & 3.40 & 0.80 \\
\hline Heart trouble & 520 & & 12.10 & 29 & & 17.20 & 0.41 \\
\hline Head injury & 520 & & 4.60 & 29 & & 6.9 & 0.43 \\
\hline
\end{tabular}


Table 3. Cognitive screening measures

\begin{tabular}{|c|c|c|c|c|c|}
\hline & \multicolumn{2}{|c|}{ Normal } & \multicolumn{2}{|c|}{ MCI } & \multirow[t]{2}{*}{$\mathrm{p}$} \\
\hline & $\mathrm{n}$ & mean $(\mathrm{SD})$ & $\mathrm{n}$ & mean $(\mathrm{SD})$ & \\
\hline MMSE & 520 & $29.15(1.43)$ & 29 & $27.37(2.07)$ & 0.00 \\
\hline Reaction time simple (mean) & 507 & $0.25(0.05)$ & 28 & $0.28(0.09)$ & 0.00 \\
\hline Reaction time choice (mean) & 505 & $0.31(0.04)$ & 28 & $0.33(0.08)$ & 0.10 \\
\hline SDMT total score & 520 & $50.17(9.31)$ & 29 & $44.03(10.29)$ & 0.00 \\
\hline Immediate recall score & 522 & $7.19(2.25)$ & 29 & $3.51(1.80)$ & 0.00 \\
\hline Delayed recall score & 522 & $6.25(2.49)$ & 29 & $2.41(1.82)$ & 0.00 \\
\hline Spot-the-word & 513 & $51.93(6.12)$ & 27 & $45.92(5.87)$ & 0.00 \\
\hline \multicolumn{6}{|l|}{ Purdue Pegboard test } \\
\hline Dominant hand & 518 & $13.35(2)$ & 29 & $12.27(2.54)$ & 0.00 \\
\hline Non-dominant hand & 514 & $12.88(1.92)$ & 29 & $11.58(2.09)$ & 0.00 \\
\hline Both hands & 515 & $10.52(1.67)$ & 29 & $9.20(2.02)$ & 0.00 \\
\hline
\end{tabular}

SDMT $=$ Symbol-Digit Modalities Test.

Table 4. Results of logistic regression analysis predicting MCI from age, gender, years of education, depression and physical disability

\begin{tabular}{lll}
\hline Predictor variables & $\begin{array}{l}\text { Association with MCI } \\
\text { OR }(95 \% \mathrm{CI})\end{array}$ & $\mathrm{p}$ value \\
\hline Age & $1.04(0.79-1.36)$ & 0.77 \\
Years of education & $0.77(0.67-0.89)$ & 0.00 \\
Goldberg depression score & $1.21(1.01-1.44)$ & 0.03 \\
Physical disability & $0.99(0.94-1.03)$ & 0.73 \\
Gender & $2.04(0.90-4.62)$ & 0.08 \\
\hline
\end{tabular}

${ }^{1}$ A higher score means less disability.

shows the results of regression using MRI measures as predictor variables after controlling for age, gender, education, physical disability and depression. To our surprise, none of the variables were significant.

\section{Discussion}

As would be expected, MCI was associated with poor performance on all cognitive measures. The only predictors of MCI were presence of depression and fewer years of education. None of the structural brain variables predicted MCI. This finding differs from those of several other studies. In the Cardiovascular Health Study Cognition Study, while there were associations between MCI and neuroradiological findings (white matter lesions, ventricular volume, cortical atrophy and MRI-identified infarcts), these associations were not significant except
Table 5. Results of logistic regression analysis predicting MCI from anterior and mid-ventricular ventricle-to-brain ratios (VBRs), cortical atrophy measures, hippocampal volumes, amygdala volumes and WMHs after adjusting for age, gender, year of education, depression and physical disability

\begin{tabular}{lll}
\hline Predictor variables & $\begin{array}{l}\text { Association with } \\
\text { MCI, OR (95\% CI) }\end{array}$ & \\
& p value \\
\hline Anterior VBR & $0.96(0.81-1.14)$ & 0.67 \\
Mid-ventricular VBR & $1.02(0.93-1.12)$ & 0.55 \\
Frontal lobe atrophy & $0.58(0.23-1.41)$ & 0.23 \\
Mid-parietal lobe atrophy & $0.43(0.09-1.93)$ & 0.27 \\
Mid-temporal lobe atrophy & $2.13(0.89-5.10)$ & 0.08 \\
Total intracranial volume & $0.99(0.98-1.00)$ & 0.38 \\
Total hippocampal volume & $1.19(0.56-2.53)$ & 0.64 \\
Left hippocampal volume & $1.63(0.37-7.11)$ & 0.51 \\
Right hippocampal volume & $1.18(0.28-4.86)$ & 0.81 \\
Total amygdala volume & $1.09(0.27-4.43)$ & 0.90 \\
Left amygdala volume & $1.60(0.14-18.34)$ & 0.70 \\
Right amygdala volume & $0.78(0.05-10.91)$ & 0.86 \\
Total brain WMH score & $1.06(0.65-1.70)$ & 0.80 \\
Total deep white matter score & $1.09(0.53-2.25)$ & 0.80 \\
Total deep gray matter score & $1.06(0.56-1.99)$ & 0.84 \\
Total periventricular white matter & & \\
\multicolumn{1}{c}{ score } & $1.00(0.35-2.84)$ & 0.85 \\
\hline
\end{tabular}

MRI-defined infarcts in the logistic regression analysis [32]. Cerebrovascular diseases were particularly associated with MCI subjects in the above study, and the mean age for MCI subjects was 75 years, much older than our sample. They also found an association with lower education and depression very similar to our findings. The Leipzig Longitudinal Study of the Aged looked at the as- 
sociation of hippocampal volumes in $75+$ subjects with MCI and found that volumetric measures of hippocampus were sensitive in discriminating subjects with questionable dementia from subjects with normal cognition and mild dementia [12]. In a longitudinal study, the Amsterdam Study of the Elderly group demonstrated that the combination of memory function and the volumes of hippocampus or the parahippocampal gyrus or the medial temporal lobe atrophy score at baseline increased the accuracy of the prediction of the clinical diagnosis of Alzheimer's disease [11]. The age range of their subjects was 65-85 years. The National Heart, Lung and Blood pressure Institute Twin Study, using a longitudinal design, showed that elevated mid-life blood pressures, and the resulting increased WMHs, increased the risk for $\mathrm{MCI}$ in a group of community-dwelling older men [33]. When the subjects were scanned in this study, they were between 67 and 81 years old.

A likely explanation for the negative findings in our study is the lower age range. Our study is the first one to report on MRI correlates in a middle-aged cohort with a very narrow age range using an epidemiological design. In this age range, the risk of developing dementia is low. This low base rate would affect the validity of the MCI diagnoses even if there was good sensitivity and specificity for predicting future dementia. For example, if the sensitivity and specificity of the MCI diagnosis is $80 \%$ and the base rate of incipient dementia is $1 \%$, then the positive predictive value will only be $4 \%$. If the base rate rises to $5 \%$, the positive predictive value is still only $23 \%$. In other words, MCI diagnostic criteria that might work reasonably well in a high-risk sample, such as patients in a memory clinic or a very elderly community sample, will work poorly in a low-risk community sample.

We found depression was strongly associated and predicted MCI. This finding is consistent with other studies $[5-7,34]$. There may be several reasons for this association. It may be that depression is causing the cognitive impairment or depression and cognitive impairment share a common biological mechanism.

Our study had a number of strengths. Firstly, the sample from which we screened the MCI subjects was large and comprised fairly representative community-dwelling individuals who had been randomly selected for participation. Secondly, we used most of the structural MRI measures and we validated the manual WMHs rating with automated method. Thirdly, we controlled for the potential confounders particularly, age, gender, education, physical health and depression.
The study had a number of limitations. Firstly, it was cross-sectional. Longitudinal follow up will determine the reliability of these diagnoses and at what age structural brain indices become predictive of MCI. We will also be able to reanalyze the data at a later stage, defining MCI retrospectively as an evolution towards dementia, and reexamining the clinical and imaging correlates in this first wave. Secondly, although the narrow age range of the study permits the ruling out of spurious statistical associations, it had the disadvantage that we were unable to examine the relationships over a wider age span. A third limitation was the non-inclusion of a number of potential participants. We acknowledge that some individuals refused to participate, and these were more likely to be older or cognitively impaired, thus making our sample healthier than a truly representative one. This limitation is common to all studies involving voluntary participation of individuals with no financial remuneration.

In our opinion, the main reason for the lack of association with MRI variables is that we assessed relatively young and healthy MCI subjects. As age is a major factor that determines brain atrophy and increased WMH load, we hypothesize that these changes had not yet occurred in our sample. However, it is possible that changes occurred in variables not included in this study, such as the entorhinal cortices. It is important to note that depression is a major predictor of MCI even at this early stage, and perhaps recognition and treatment of depression is a more appropriate target for intervention in younger MCI patients.

\section{Acknowledgement}

This study was supported by NHMRC program grant 179805 and NHMRC project grant 157125 . We thank the following for their contribution to the PATH Through Life Project: Trish Jacomb, MSc; Karen Maxwell; June Cullen, RN; Jeremy Price, FRACR; Greg de Plater, PhD; Bryan Rodgers, PhD, and the PATH interviewing team. 


\section{References}

1 Petersen RC, Doody R, Kurz A, Mohs RC, Morris JC, Rabins PV, et al: Current concepts in mild cognitive impairment. Arch Neurol 2001;58:1985-1992.

-2 Petersen RC, Smith GE, Waring SC, Ivnik RJ, Tangalos EG, Kokmen E: Mild cognitive impairment: Clinical characterization and outcome. Arch Neurol 1999;56:303-308.

3 Luis CA, Loewenstein DA, Acevedo A, Barker WW, Duara R: Mild cognitive impairment: Directions for future research. Neurology 2003;61:428-444.

-4 Ritchie K, Artero S, Touchon J: Classification criteria for mild cognitive impairment: A population-based validation study. Neurology 2001;56:37-42.

-5 Leketos CG, Lopez O, Jones B, Fitzpatrick AL, Breitner J, DeKosky S: Prevalence of neuropsychiatric symptoms in dementia and mild cognitive impairment: Results from the Cardiovascular Health Study. JAMA 2002;288: 1475-1483.

-6 Palmer K, Forsell Y, Fratiglioni L: Psychiatric symptoms in elderly persons with mild cognitive impairment. Data from a cross-sectional study. Acta Neurol Scand 2003;107:25-28.

7 Gabryelewicz T, Styczynska M, Pfeffer A, Wasiak B, Barczak A, Luczywek E, Androsiuk W, Barcikowska M: Prevalence of major and minor depression in elderly persons with mild cognitive impairment-MADRS factor analysis. Int J Geriatr Psychiatry 2004;19:11681172.

-8 De Santi S, de Leon MJ, Rusinek H, Convit A, Tarshish CY, Roche A, et al: Hippocampal formation glucose metabolism and volume losses in MCI and AD. Neurobiol Aging 2001;22: 529-539.

-9 Jack CR Jr, Shiung MM, Gunter JL, O'Brien PC, Weigand SD, Knopman DS, et al: Comparison of different MRI brain atrophy rate measures with clinical disease progression in AD. Neurology 2004;62:591-600.

10 Wolf H, Jelic V, Gertz H-J, Nordberg A, Julin P, Wahlund L-O: A critical discussion of the role of neuroimaging in mild cognitive impairment. Acta Neurol Scand 2003;107:52-76.

11 Visser PJ, Scheltens P, Verhey FR, Schmand B, Launer LJ, Jolles J, et al: Medial temporal lobe atrophy and memory dysfunction as predictors for dementia in subjects with mild cognitive impairment. J Neurol 1999;246:477485.
12 Wolf H, Grunwald M, Kruggel F, Riedel-Heller SG, Angerhofer S, Hojjatoleslami A, et al: Hippocampal volume discriminates between normal cognition; questionable and mild dementia in the elderly. Neurobiol Aging 2001; 22:177-186.

13 Folstein MF, Folstein SE, McHugh PR: MiniMental State: A practical method for grading the cognitive state of patients for the clinician. J Psychiatr Res 1975;12:189-198.

14 Delis DC, Kramer JH, Kaplan EF: California Verbal Learning Test. San Antonio, Psychological Corporation, 1987.

15 Smith A: Symbol Digit Modalities Test (TMT) Manual, rev ed. Los Angeles, Western Psychological Services, 1982.

16 Tiffin J: Purdue Pegboard. Examiners Manual. Rosemont, London House, 1968.

17 Christensen H, Jorm AF, Henderson AS, et al: The relationship between health and cognitive functioning in a sample of elderly people in the community. Age Ageing 1994;23:204-212.

18 Reitan RM: Validity of the Trail Making Test as an indicator of organic brain syndrome. Percept Mot Skills 1958;8:271-276.

19 Borkowski J, Benton, AL, Spreen O: Word fluency and brain damage. Neuropsychologia 1967;5:135-140.

20 Borod JC, Goodglass H, Kaplan E: Normative data on the Boston Diagnostic Naming Test. J Clin Neuropsychol 1980;2:209-216.

21 Kaplan EF, Goodglass H, Weintraub S: The Boston Naming Test. Boston, Psychological Corporation, 1991.

22 Morris JC, Heyman A, Mohs RC: The consortium to establish a registry for Alzheimer's Disease (CERAD). 1. Clinical and Neuropsychological Assessment of Alzheimer's disease. Neurology 1989;39:1159-1165.

23 Lezak MD: Neuropsychological Assessment, ed 3. Oxford, Oxford University Press, 1995.

24 Morris JC: The clinical dementia rating (CDR): current version and scoring rules. Neurology 1993;43:2412-2414
25 Kumar R, Dear KB, Christensen H, Ilschner S, Jorm AF, Meslin C, Rosenman SJ, Sachdev PS: Prevalence of mild cognitive impairment in 60- to 64-year-old community-dwelling individuals: the Personality and Total Health through Life 60+ Study. Dement Geriatr Cogn Disord 2005; 19:67-74.

26 Goldberg D, Bridges K, Duncan-Jones P, Grayson D: Detecting anxiety and depression in general medical settings. BMJ 1988;297: 897-899.

27 Spitzer R, Kroenke K, Williams JWB: Validation and utility of a self-report version of PRIME-MD: the PHQ primary care study. JAMA 1999;282:1737-1744.

-28 Ware J, Kosinski M, Keller SD: A12-item short-form health survey: Construction of scales and preliminary tests of reliability and validity. Med Care 1996;34:220-233.

29 Victoroff J, Mack WJ, Grafton ST, Schreiber SS, Chui HC: A method to improve interrater reliability of visual inspection of brain MRI scans in dementia. Neurology 1994;44:22672276.

30 Watson C, Jack CR Jr, Cendes F: Volumetric magnetic resonance imaging. Clinical applications and contributions to the understanding of temporal lobe epilepsy. Arch Neurol 1997; 54:1521-1531.

-31 Fazekas F, Barkhof F, Wahlund LO, Pantoni L, Erkinjuntti T, Scheltens P, Schmidt R: CT and MRI rating of white matter lesions. Cerebrovasc Dis 2002;13:31-36.

- 32 Lopez O, Jagust WJ, Dulberg C, Becker JT, DeKoski ST, Fitzpatrick A, Breitner J, Lykestos C, Jones B, Kawas C, Carlson M, Kuller LH: Risk factors for mild cognitive impairment in the Cardiovascular Health study Cognition Study. Arch Neurol 2003;60:1394-

-33 DeCarli C, Miller BL, Swan GE, Reed T, Wolf PA, Carmelli D: Cerebrovascular and brain morphologic correlates of mild cognitive impairment in the National Heart, Lung, and Blood Institute Twin Study. Arch Neurol 2001; 58:643-647.

34 Chan D-C, Kasper JD, Black BS, Rabins PV: Prevalence and correlates of behavioral and psychiatric symptoms in community-dwelling elders with dementia or mild cognitive impairment: the Memory and Medical Care Study. Int J Geriatr Psychiatry 2003;18:174-182. 